\title{
Methyl jasmonate elicitation of common bean seedlings induces nucleotidase activity and the expression of several nucleotidase genes in radicles
}

\author{
G. GALVEZ-VALDIVIESO (D, M. GARMENDIA-CALVO, M. PINEDA, and P. PIEDRAS* \\ Departamento de Botánica, Ecología y Fisiología Vegetal, Campus de Rabanales, Edif. Severo Ochoa, \\ Universidad de Córdoba, 14071-Córdoba, Spain \\ *Corresponding author: E-mail: bb2pimop@uco.es
}

\begin{abstract}
Nucleotides are the basic elements of the genetic material, participate in bio-energetic processes, are cofactors and components of secondary metabolites, etc. Nucleotide hydrolases (nucleotidases) are phosphatases that remove the 5 '-phosphate group from the nucleotides and play a crucial role in nucleotide metabolism. In this study, genes encoding putative nucleotidases in Phaseolus vulgaris have been identified, and the effect of methyl jasmonate (MeJA) on both nucleotidase activity and gene expression has been addressed. The predicted nucleotidase peptides include the conserved domains characteristic of the haloacid dehalogenase-like hydrolase superfamily. The analysis of the expression of the 11 identified genes in radicles of common bean seedlings elicited with MeJA showed that 3 of them are highly induced by this phytohormone in a dose-dependent manner. Nucleotidase activity in radicles from MeJA treated plants was higher than in not elicited seedlings, and this induction was observed with all the nucleotides assayed (mono-, di- or triphosphate) and with purine or pyrimidine nucleotides. MeJA is involved in biotic and abiotic stress, and the induction of nucleotide metabolism in response to this treatment suggests a relevant role for nucleotides in the seedlings response to unfavourable conditions.

Keywords: haloacid dehalogenase-like hydrolases superfamily, phosphatases, stresses.
\end{abstract}

\section{Introduction}

Nucleotides are essential molecules with implications for vital processes such as plant germination, as well as development and growth. Nucleotides are essential in the storage and recovery of genetic information acting as structural elements for the formation of DNA and RNA, act as a source of energy at the cellular level, and form parts of different cellular components such as vitamins (vitamin B12), hormones (such as cytokinin), or enzyme cofactors (such as $\mathrm{NAD}^{+}$) (Zrenner et al. 2006, Haferkamp et al. 2011). In addition, nucleotides have phosphate in their molecule, which can be transferable to other molecules (Zrenner et al. 2006).
Purine nucleotides have an additional key role in ureidic legumes, such as common bean or soybean. Ureidic legumes mobilize most of the nitrogen fixed in root nodules to the aerial organs of the plants in the form of ureides when they grow under nitrogen-fixing conditions (Todd et al. 2006). Ureides (allantoate and allantoin) are organic substances formed by the oxidation of purines with an elevated $\mathrm{N}$ : $\mathrm{C}$ ratio $(1: 1)$. In addition to their role as transporters of fixed nitrogen in ureidic legumes, ureides may be involved in other processes related to the storage and mobilization of nitrogen, and a protective role for ureides against reactive oxygen species has been suggested in stress situations (Watanabe et al. 2014), although this protective role could be related with

$\overline{\text { Received }} 17$ November 2020, last revision 2 March 2021, accepted 4 March 2021.

Abbreviations: HAD - haloacid dehalogenase-like hydrolases; MeJA - methyl jasmonate; MES - 2-(N-morpholino)ethanesulfonic acid; NTD - nucleotidase; p-NPP - para-nitrophenyl phosphate; TES - N-[Tris(hydroxymethyl)methyl]-2-aminoethanesulfonic acid.

Acknowledgements: This work was funded by the Ministerio de Economía y Competitividad (AGL2015-69554) and the Plan Andaluz de Investigación (BIO115). The authors thank Marta Robles for her technical assistance and Manuel Sánchez for his collaboration in database analysis.

Conflict of interest: The authors declare that they have no conflict of interest. 
its ability as nitrogen reservoir rather than stress protectant (Soltabayeva et al. 2018). In this way, legume seedlings show differences in antioxidant enzymatic activities in relation to their behaviour as ureidic or amidic legumes (Quiles et al. 2019). Induction of ureide metabolism has been reported during germination and post-germinative development in common bean (Quiles et al. 2009), and ureide accumulation has been described during darkinduced bean leaf senescence (Lambert et al. 2017) and nitrogen starvation in Arabidopsis (Melino et al. 2018) associated, in both cases, with nucleic acid catabolism. The nucleic acid metabolism could be used to mobilize nitrogen and phosphorus during senescence (Lambert et al. 2016, 2017) and the importance of their mobilization during senescence has been reported (Soltabayeva et al. 2018).

The metabolic pathways of nucleotides have been studied fundamentally in animals, due to the importance of nucleotide metabolism in the investigation of genetic diseases and in cancer research (Camici et al. 2019). In plants, the knowledge about the enzymes involved in nucleotide metabolism is more limited. This can be due to the numerous reactions that participate in the de novo synthesis, salvage, and degradation (Zrenner et al. 2006). Both, the salvage and de novo synthesis pathways merge in the formation of nucleosides monophosphate. The catabolic pathway begins with the cleavage of the 5 '-phosphate group, a reaction catalysed by a phosphatase that hydrolyses the nucleotides into nucleosides. However, it has not been elucidated if this reaction is carried out by different enzymes or if there are specific nucleotidases for each. A phosphatase with elevated affinity for nucleoside monophosphate was purified from embryonic axes of common bean (Cabello-Díaz et al. 2012), and the gene encoding the protein was identified (Cabello-Díaz et al. 2015), as well as another gene whose expression is higher in the radical nodules where ureides are synthesized (Galvez-Valdivieso et al. 2020).

Jasmonates are phytohormones that regulate various physiological processes of plant development including root growth, flowering, and leaf senescence (Huang et al. 2017), and participate in the activation of the defence responses of plants to abiotic stress and pathogenic attacks (Dar et al. 2015, Raza et al. 2020, Wang et al. 2020). Jasmonates derive from lipids and include jasmonic acid (JA) and its derivatives, jasmonate iso-leucine conjugate and methyl jasmonate (MeJA) (Wasternack et al. 2018). The tissue damaged during wounding results in the release of cellular components to the extracellular space, including nucleic acids and nucleotides. Furthermore, extracellular ATP (eATP) is an emerging signalling molecule in plant metabolism that exerts its function acting synergistically with jasmonate (Tripathi et al. 2018, Pietrowska-Borek et al. 2020). To maintain its function, eATP homeostasis must be controlled and phosphatases should play a crucial role in this process (Clark et al. 2011).

To better understand the nucleotide metabolism in plants and its possible relationship with the response to stress, we have identified genes that code for nucleotidase members of the haloacid dehalogenase-like hydrolases
(HAD) superfamily in Phaseolus vulgaris. Furthermore, we analysed their expression pattern in common bean radicles in response to MeJA treatment.

\section{Materials and methods}

Plants and growing conditions: Phaseolus vulgaris L. cv. Great Northern seeds were sterilized and germinated on Petri dishes as previously described (Lambert et al. 2014). After $5 \mathrm{~d}$ from the start of the imbibition, the seedlings were placed in new plates with filter paper moistened with distilled water (control treatment), or with the indicated concentration of MeJA and they were maintained in the growth chamber for $24 \mathrm{~h}$. After that time, the seedlings were separated into radicles, the aerial part of embryonic axes and cotyledons and immediately frozen in liquid nitrogen.

Preparation of crude extracts: The plant material (approximately $100 \mathrm{mg}$ ) was pulverized with mortar in liquid nitrogen. The pulverized material was mixed with 4 volumes of extraction buffer consisting of $50 \mathrm{mM}$ $\mathrm{N}$-[Tris(hydroxymethyl)methyl]-2-aminoethanesulfonic acid (TES; $\mathrm{pH} 7.0$ ) and $0.15 \% \mathrm{w} / \mathrm{v}$ sodium deoxycholate (DOC). Once homogenized, it was centrifuged at $24000 \mathrm{~g}$ and $4{ }^{\circ} \mathrm{C}$ for $10 \mathrm{~min}$. Finally, the supernatant was collected and considered as a crude extract.

Enzymatic activities determination: Phosphatase activities were assayed by monitoring the phosphate concentration in reaction mixtures as previously described (Cabello-Diaz et al. 2012). Total phosphatase activity was assayed in a standard reaction mixture containing $50 \mathrm{mM}$ 2-(N-morpholino)ethanesulfonic acid (MES$\mathrm{HCl})$ buffer ( $\mathrm{pH} 5.5), 2 \mathrm{mM}$ para-nitrophenyl phosphate (pNPP) as substrate and an adequate amount of crude extract. Nucleotidase activity was determined in a mixture containing $50 \mathrm{mM}$ MES-HCl buffer (pH 5.5) or $50 \mathrm{mMTES}$ $\mathrm{NaOH}$ (pH 7), $1 \mathrm{mM} \mathrm{MgCl}$, 2 mM nucleotide indicated as substrate, $5 \mathrm{mM}$ molybdate and an adequate amount of enzyme crude extract. The reactions were initiated by the addition of the enzyme crude extract and were performed at $37{ }^{\circ} \mathrm{C}$. Aliquots of $0.2 \mathrm{~cm}^{3}$ were extracted at various time points and phosphate concentration was determined (Cabello-Diaz et al. 2012).

RNA isolation, cDNA synthesis and real-time PCR: Total RNA isolation, cDNA synthesis, and real-time quantitative PCR (qPCR) were performed as previously described (Galvez-Valdivieso et al. 2020) using the specific primers indicated in Table 1 Suppl. Results were normalised using the geometric mean of ubiquitin and actin-2 using the $2^{-\Delta \mathrm{CT}}$ or $2^{-\Delta \Delta \mathrm{CT}}$ method (Livak and Schmittgen 2001). The specificity of the pair of primers was verified by real-time PCR and sequencing of the products and following the amplicon dissociation curves.

Analytical Determination: Protein content in the crude extracts was determined by the protein-dye binding 
method (Bradford 1976) using the Bio-Rad (Hercules, USA) reactive substance and with bovine serum albumin as standard. Total ureides were determined by a colorimetric method as described by Quiles et al. (2019).

Sequences analysis: The sequences were obtained from the Phytozome v. 12.1 (https://phytozome.jgi.doe.gov/pz/ portal.html\#) and NCBI (https://blast.ncbi.nlm.nih.gov// databases. The alignment of sequences was performed with MegAlign from DNASTAR Lasergene 7.0.0. Specific primers were designed using Primer 3 plus (http://www. bioinformatics.nl/cgi-bin/primer3plus/primer3plus.cgi) (Untergasser et al. 2007). The phylogenetic tree was obtained after the alignment of the predicted proteins with the algorithms MUSCLE (Edgar 2004) from the program MEGA X 10.0.5 (Kumar et al. 2018). The phylogenetic tree was elaborated with the method neighbour-joining (NJ) (Saitou and Nei 1987) with a bootstrap value of 1000 replicates. Subcellular localization of the predicted proteins was performed using DeepLoc-1.0 eukaryotic protein subcellular localization predictor (http://www. cbs.dtu.dk/services/DeepLoc/cite.php) (Armenteros et al. 2017). The platform New Place (https://www.dna.affrc. go.jp/PLACE/?action=newplace)_(Higo 1998) was used to analyse the promoter regions of the genes.

Statistical analyses: All results are means of three biological replicates. The analyses performed are indicated in the legend to figures. Statistical analyses were performed with SPSS Statistics, version 25.

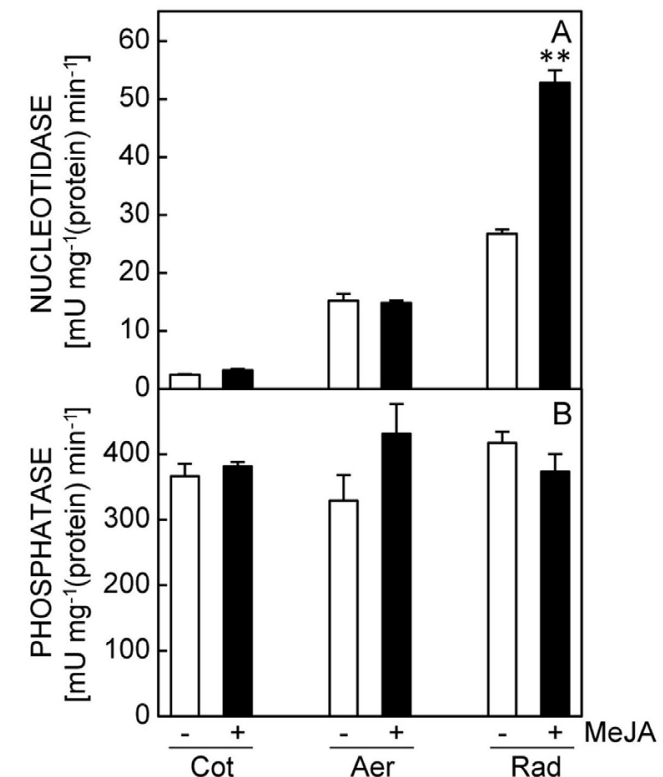

Fig. 1. Nucleotidase $(A)$ and total acid phosphatase $(B)$ in cotyledons (Cot), aerial part (Aer), or radicles (Rad) of French bean seedlings in the absence of MeJA (-) or treated for $24 \mathrm{~h}$ with $50 \mu \mathrm{M}$ MeJA $(+)$. Nucleotidase and total acid phosphatase were determined with inosine monophosphate (IMP) and paranitrophenyl phosphate (pNPP) as substrates, respectively. Means \pm SEs of three biological replicates. A $t$-test was carried out and a significant value at $P \leq 0.001$ is indicated by two asterisks.

\section{Results}

The effect of methyl jasmonate on nucleotidase and phosphatase activities was analysed in cotyledons, axes, and radicles of 6-d-old seedlings after $24 \mathrm{~h}$ of treatment with $50 \mu \mathrm{M}$ of MeJA. Nucleotidase activity was lower in cotyledons with respect to axes and radicles (Fig. 1A). MeJA treatment did not produce any relevant effect on the nucleotidase activity in cotyledons and axes (Fig. 1A) while it caused an increase of nearly $100 \%$ in radicle nucleotidase activity. The total phosphatase activity was much higher than nucleotidase activity in the three tissues, and no significant differences in activity were detected after the MeJA treatment (Fig. 1B). Ureide content in common bean seedlings parts was not affected by MeJA treatment (Fig. 1 Suppl.).

To identify the putative gene or genes responsible for the increase in nucleotidase activity in radicles after MeJA treatment a search in the database was performed. The sequences coding for putative nucleotidases $P v N T D 1$ (Cabello-Diaz et al. 2015) and PvNTD2 (Galvez-Valdivieso et al. 2020) were used to search in the Phytozome and NCBI databases. In this way, 9 additional sequences were identified, which were named from PvNTD3 to $P v N T D 11$ and their respective accession numbers in the Phytozome database are indicated in the legend to Fig. 2. All the sequences belong to the subfamily IIIB of HAD superfamily of phosphatases and are grouped in the family PF03767 in the Pfam database (https://pfam.xfam.org/). The predicted proteins encoded by these genes would range between 251 and 312 amino acids. The alignment of these sequences as well as the domains shared by the acid phosphatases of the HAD family (Burroughs et al. 2006) is shown in Fig. 2. A crucial nucleophilic asparagine and the $\operatorname{DxDx}(\mathrm{T} / \mathrm{V})(\mathrm{L} / \mathrm{V})$ consensus sequence form part of the HAD signature motif $\mathrm{I}$, and this motif together with motif IV are involved in the coordination of $\mathrm{Mg}^{2+}$ in the active site. Motif II consists in the consensus sequence $\mathrm{hhh}(\mathrm{S} / \mathrm{T})$, where $h$ represents a hydrophobic residue and $\mathrm{S} / \mathrm{T}$ amino acids serin and threonin. The degree of conservation of Motif III is reduced when compared to the other motifs, with a lysin residue mostly conserved. Motif IV generally contains the consensus sequence $(\mathrm{G} / \mathrm{S})(\mathrm{D} / \mathrm{S}) \mathrm{x}_{3-4}(\mathrm{D} / \mathrm{E})$, but,

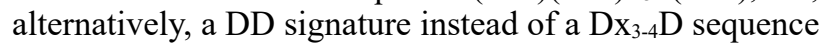
has been also observed (Seifried et al. 2013).

A phylogenetic tree was elaborated, and 2 differentiated groups were observed (Fig. 3). PvNTD4 and PvNTD6 are in a separate branch from all the other sequences. The other branch is divided into two groups, with PvNTD1, PvNTD2, PvNTD9, PvNTD10, and PvNTD11 in the same group. The two previously characterized genes $(P v N T D 1$ and $P v N T D 2)$ are in the same branch, and the other three are in a separate one.

To elucidate the possible location of the proteins that encode these genes, analysis of the amino acid sequences was carried out using DeepLoc-1.0 (Table 1). The results suggest that most of the proteins could be extracytosolic, located either in the extracellular space (PvNTD1, PvNTD2, PvNTD3, PvNTD9, PvNTD10, and PvNTD11), or in organelles such as the lysosome or the vacuole, 

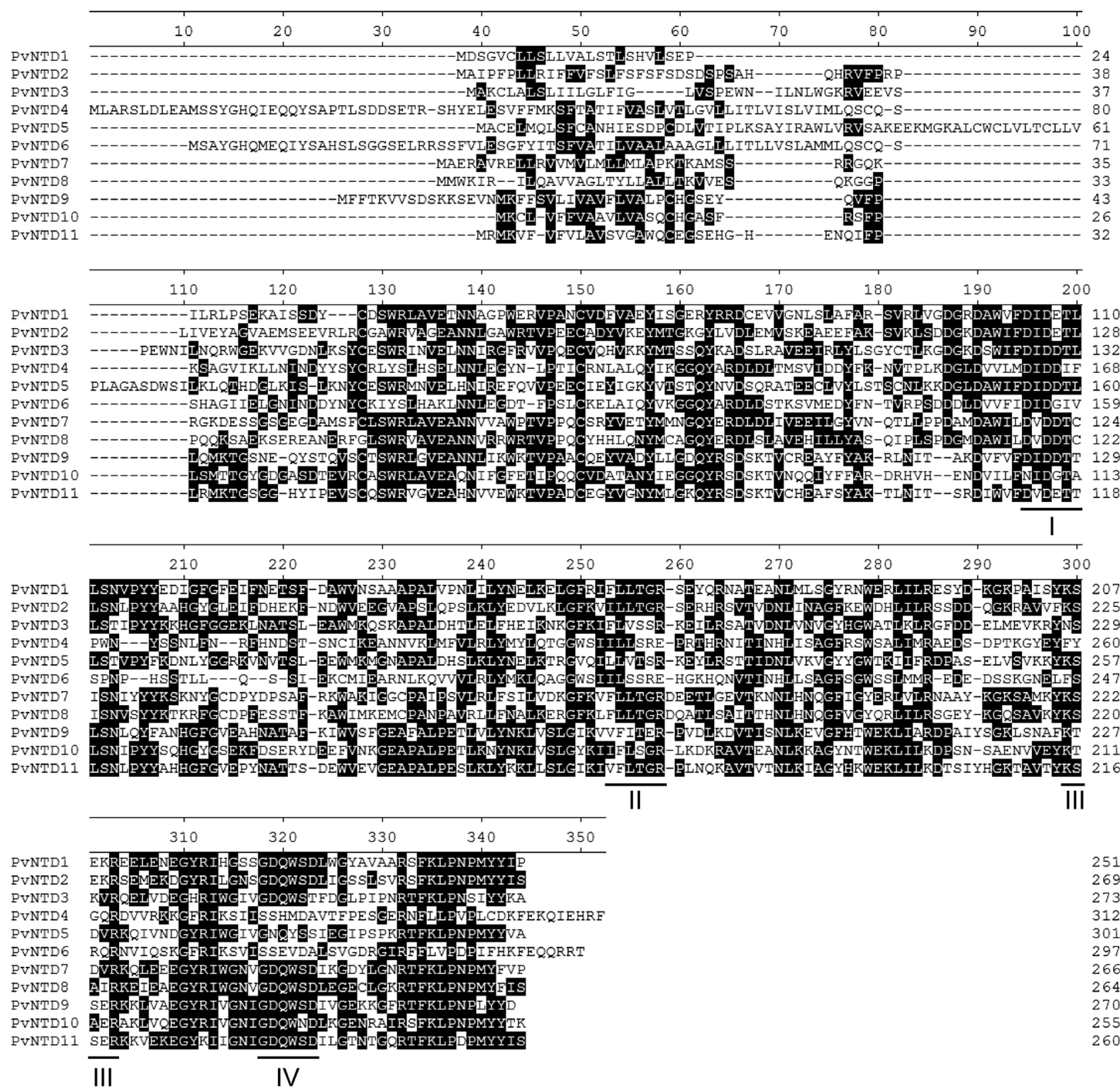

Fig. 2. Alignment of amino acid sequences of French bean genes belonging to pfam family PF03767. The corresponding accession numbers for each gene are PvNTD1 (Phvul.004G174200), PvNTD2 (Phvul.011G182400), PvNTD3 (Phvul.001G164000), PvNTD4 (Phvul.001G240100), PvNTD5 (Phvul.007G270800), PvNTD6 (Phvul.008G227000), PvNTD7 (Phvul.010G058800), PvNTD8 (Phvul.010G059000), PvNTD9 (Phvul.010G144200), PvNTD10 (Phvul.010G144300), PvNTD11 (Phvul.010G144600). The amino acids identical to the consensus sequence are shadowed in black. The different motifs of the haloacid dehalogenase (HAD) superfamily (Burroughs et al. 2006) are underlined. The sequences were aligned using the ClustalW method.

endoplasmic reticulum, or in the membrane (PvNTD4, PvNTD5, PvNTD6, PvNTD7, and PvNTD8). Of all the sequences analysed, PvNTD4 and PvNTD6 appear to be membrane proteins, while all the others appear to be soluble.

Since the nucleotidase activity changed in response to MeJA in radicles (Fig. 1), the expression of the 11 identified genes from common bean was analysed in radicles from both control and $50 \mu \mathrm{M}$ MeJA treated seedlings (Fig. 4). All the genes were detected in non-treated radicles and the expressions were very variable, being PvNTD5 (relative gene expression of 0.0002 ) and PvNTD10 (relative gene expression of 0.78 ) the genes with the lowest and highest transcriptions, respectively (Fig. 4). Treatment with MeJA for $24 \mathrm{~h}$ induced the expression of PvNTD9, PvNTD10, and $P v N T D 11$, whereas did not significantly affect the expressions of the other genes (Fig. 4). The MeJA treatment resulted in a 4.4-, 3.2- and 14.1-fold increase in the relative expression of $P v N T D 9, P v N T D 10$, and PvNTD11, respectively. 


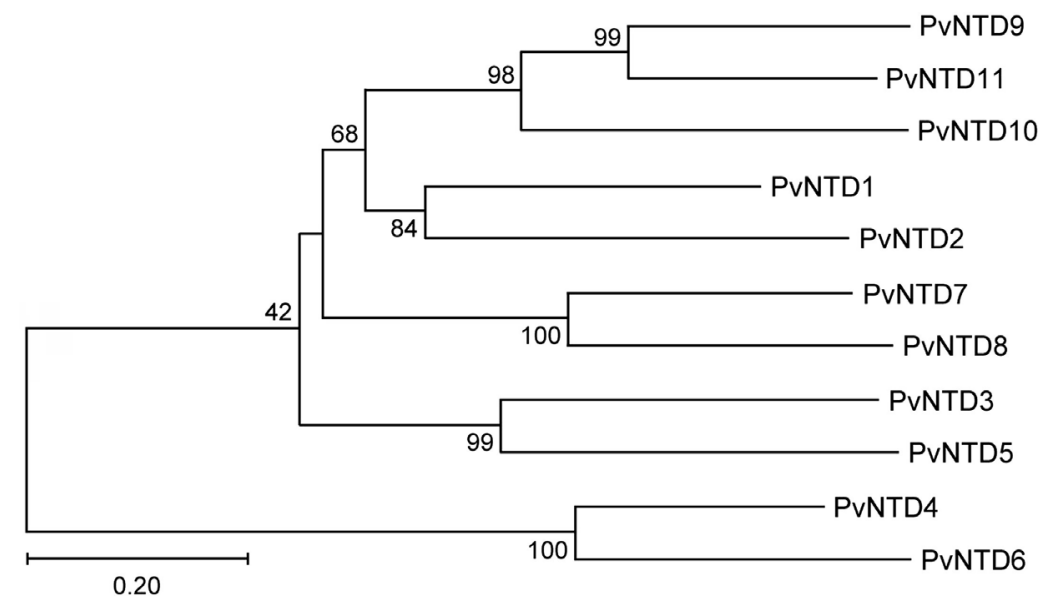

Fig. 3. Evolutionary relationships of pfam family PF03767 sequences of French bean. The tree is represented to scale. The branch lengths are in the same units as those of the evolutionary distances used to infer the phylogenetic tree. Evolutionary distances were calculated using the Poisson correction method, and are in units of the number of amino acid substitutions per spot. All ambiguous positions were eliminated for each sequence pair (pairwise remove option). The analysis was performed with the Muscle algorithm of the $M E G A X v \cdot 10.0 .5$ program.

Table 1. Subcellular localization and types of proteins predicted from the identified genes. More than a putative localization is shown when the prediction is lower than $50 \%$.

\begin{tabular}{|c|c|c|c|c|c|c|}
\hline Sequence & $\begin{array}{l}\text { Localization } \\
\text { extra cellular }\end{array}$ & lysosome/ vacuole & endoplasmic reticulum & membrane & $\begin{array}{l}\text { Type } \\
\text { soluble }\end{array}$ & membrane \\
\hline PvNTD1 & 0.844 & & & & 0.997 & \\
\hline PvNTD2 & 0.727 & & & & 0.990 & \\
\hline PvNTD3 & 0.504 & & & & 0.974 & \\
\hline PvNTD4 & & 0.239 & 0.236 & 0.325 & & 0.977 \\
\hline PvNTD5 & & 0.321 & 0.225 & & 0.591 & \\
\hline PvNTD6 & & 0.324 & & 0.325 & & 0.971 \\
\hline PvNTD7 & 0.312 & & 0.401 & & 0.896 & \\
\hline PvNTD8 & 0.321 & 0.454 & & & 0.821 & \\
\hline PvNTD9 & 0.577 & & & & 0.977 & \\
\hline PvNTD10 & 0.924 & & & & 0.999 & \\
\hline PvNTD11 & 0.733 & & & & 0.997 & \\
\hline
\end{tabular}

Since the transcriptions of PvNTD9, PvNTD10, and $P v N T D 11$ increased significantly in the presence of $50 \mu \mathrm{M}$ MeJA, the effect of different concentrations of MeJA on their expression was analysed. As shown in Fig. 5, there was a dose-dependent relation between the concentration of MeJA and the transcriptions of these 3 genes, being PvNTD11 the gene that shows the strongest response to the addition of MeJA (Fig. 5).

The region containing the 1000 nucleotides upstream from the translation start was identified in the Phytozome database, and the cis-regulatory elements of the three genes induced by MeJA were analysed. In the three promoter regions, various motifs of response to water stress, wounding, pathogens, and hormones were identified (Table 2 Suppl.). Motifs related to biotic and abiotic stresses were identified in promoter regions of these genes (Table 2 Suppl.). In two of them, PvNTD9 and $P v N T D 11$, there were motifs of a specific response to methyl jasmonate.

Since $250 \mu \mathrm{M}$ of MeJA provokes a higher increase in the expressions of $P v N T D 9,10$, and 11 , nucleotidase activity was determined in crude extracts from radicles of seedling after $24 \mathrm{~h}$ of treatment with $250 \mu \mathrm{M}$ of MeJA, using different nucleotides as substrates at both pH 5.5 and $\mathrm{pH} 7.0$ (Fig. 6). The activity in radicles of untreated seedlings was very similar with most of the substrates assayed, being generally higher at $\mathrm{pH} 7.0$ than at $\mathrm{pH}$ 5.5. Thus, at $\mathrm{pH} 5.5$, the specific activity values ranged from $37 \mathrm{mU} \mathrm{mg}^{-1}$ (protein) $\mathrm{min}^{-1}$ for AMP to $87 \mathrm{mU} \mathrm{mg}^{-1}$ (protein) $\mathrm{min}^{-1}$ for UMP, whereas at $\mathrm{pH} 7.0$ it ranged from $70 \mathrm{mU} \mathrm{mg}^{-1}$ (protein) $\mathrm{min}^{-1}$ for AMP to $139 \mathrm{mU} \mathrm{mg}^{-1}$ (protein) $\mathrm{min}^{-1}$ for ATP. Only with ADP, the activity was markedly higher, with values of 269 and $616 \mathrm{mU} \mathrm{mg}^{-1}$ (protein) $\mathrm{min}^{-1}$ at $\mathrm{pH} 5.5$ and 7.0, respectively (Fig. 6). With all nucleotides used at both pHs, the MeJA treatment resulted in a significant increase in nucleotidase activity. At both $\mathrm{pH} 5.5$ and $\mathrm{pH} 7.0$, the highest nucleotidase activity was recorded with ADP, being almost 3 times higher when the assay was performed at $\mathrm{pH} 7.0$ and with a value of $3 \mathrm{U} \mathrm{mg}^{-1}$ (protein) $\mathrm{min}^{-1}$ (Fig. 6). 


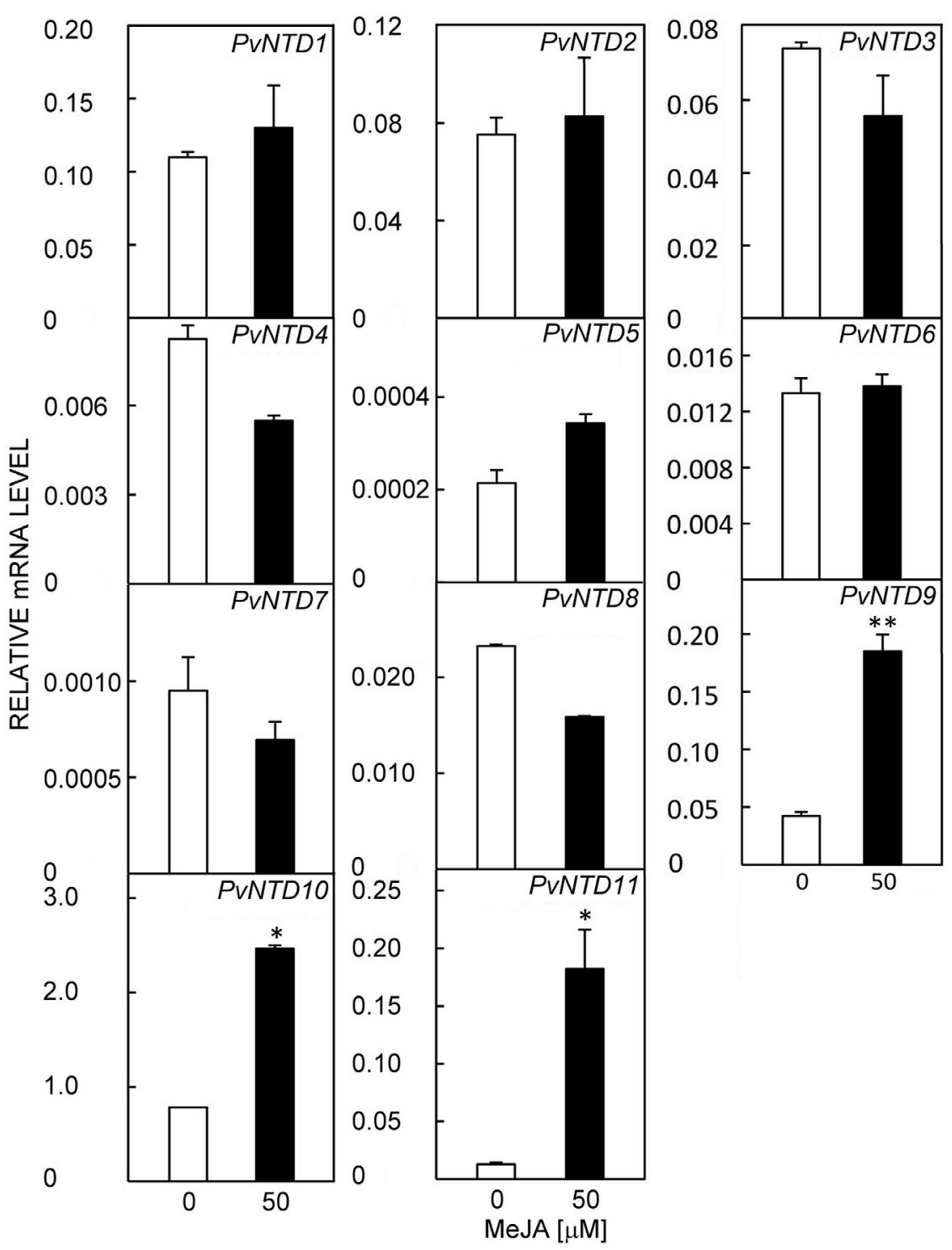

Fig. 4. Effect of MeJA on the expression of pfam family PF03767 genes in common bean radicles. Radicles of common bean at $6 \mathrm{~d}$ after the start of imbibition and incubated with the 0 or $50 \mu \mathrm{M}$ of MeJA during the last $24 \mathrm{~h}$ were analysed. Means \pm SEs of three biological replicates, with three technical replicates. Significant values according to $t$-test are indicated by asterisks $(*-P \leq 0.01, * *-P \leq 0.001)$.

\section{Discussion}

Jasmonates are phytohormones that are involved in the defence of the plant against attack by herbivores and pathogens, in tolerance to abiotic stress, and regulation of various aspects of plant physiology such as root growth, flowering, and leaf senescence (Huang et al. 2017). The application of methyl jasmonate resulted in an increase in phosphatase activity for nucleotides as substrates in the presence of molybdate in the radicles of common bean seedlings. Molybdate is an inhibitor of unspecific phosphatases (Duff et al. 1994) and therefore in these assays, the phosphatase activity should correspond to a nucleotidase (Cabello-Diaz et al. 2012). In comparison to total phosphatase activity, nucleotidase represents only a small fraction. In fact, when total phosphatase was determined in the absence of molybdate and with
pNPP as substrate, no differences were obtained after MeJA application. Induction of nucleotidase activity was observed only in radicles. The radicle is also the part of the seedling that shows changes in ribonuclease in response to salt stress (Diaz-Baena et al. 2020). Plant roots are organs with a particularly high diversity of responses to challenging environments, rearranging their development to cope with adverse situations (Gruber et al. 2013).

To determine the gene or genes responsible for the induction of nucleotidase activity in response to MeJA, nine additional genes were identified based on similarity to previously characterized $P v N T D 1$ (Cabello-Diazetal.2015) and PvNTD2 (Galvez-Valdivieso et al. 2020). All of them have the typical domains of the HAD superfamily, which groups, among other enzymes, hydrolases, phosphatases, nucleotidases and several phosphotransferases (Bogan and Brenner 2010). The catalytic action of HAD phosphatases 


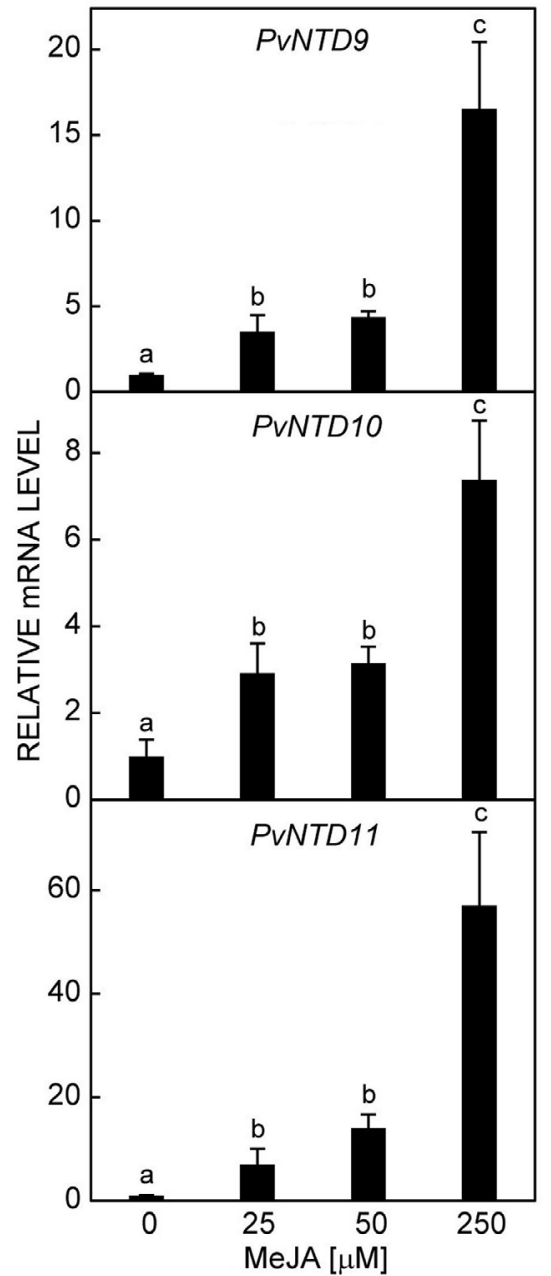

Fig. 5. Effect of MeJA concentration on the expression of $P v N T D 9, P v N T D 10$, and PvNTD11 in French bean radicles. Common bean seedlings were grown as indicated in Fig. 4 and treated for $24 \mathrm{~h}$ with $0,25,50$, and $250 \mu \mathrm{M}$ MeJA. Means \pm SEs of three independent experiments with three replicates per experiment. For each gene, the values were normalized to the values in control radicles. Significant differences according to the Tukey test are indicated with different letters $(P \leq 0.05)$.

differs from other well-known phosphatases. This group of phosphatases use an Asp residue as a nucleophile in an $\mathrm{Mg}$-dependent phosphoaspartyl transferase reaction (Seifried et al. 2013). The effect of magnesium was demonstrated for the nucleotidase PvNTD1 purified from embryonic axes from common bean, being the only cation that activated the purified enzyme (Cabello-Diaz et al. 2012). This distinctive feature of HAD phosphatases in relation to the transferase reaction is also responsible for their lack of sensitivity against the most common inhibitors of phosphatases (Seifried et al. 2013). Apart from these motifs, the sequence similarity between HAD phosphatases is very low.

The three genes that are induced in response to MeJA are in the same branch in the phylogenetic tree and they are located very close in the same chromosome in the common bean. A wound-induced gene, PtdAP1, from poplar

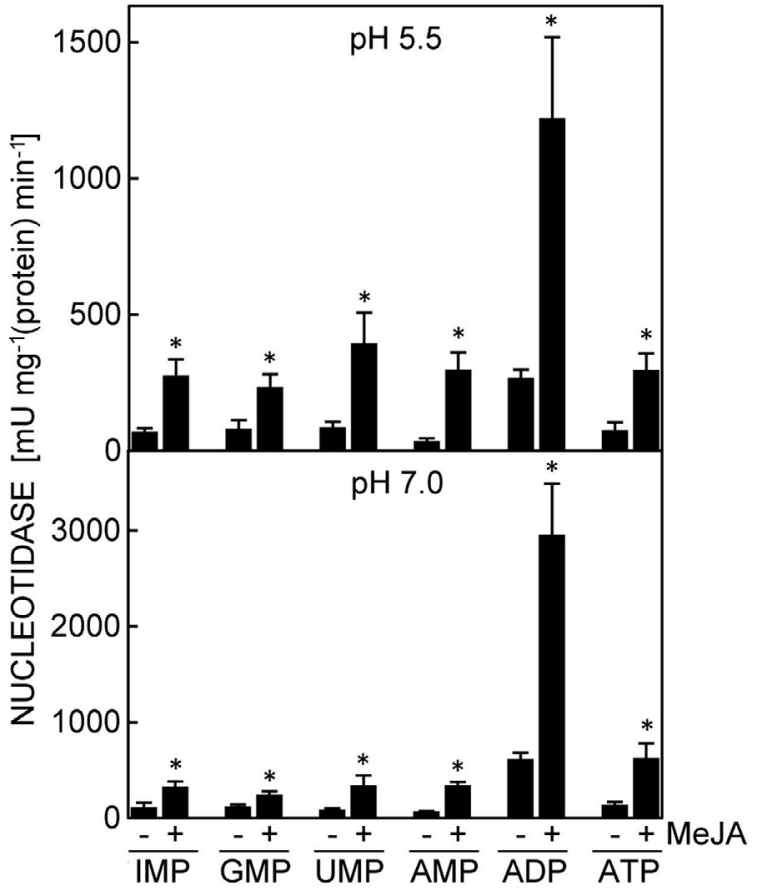

Fig. 6. Nucleotidase activity with different nucleotides at $\mathrm{pH} 5.5$ and $\mathrm{pH}$ 7.0. The activity was tested with the nucleotides indicated at both pHs in crude extracts from radicles treated with 0 or $250 \mu \mathrm{M}$ MeJA. The asterisks refer to the significant difference in nucleotidase activity between the control $(0 \mu \mathrm{M}$ MeJA $)$ and the treatment $(250 \mu \mathrm{M}$ MeJA) (Student's $t$-test; $P<0.001)$. Means \pm SEs of three biological replicates.

(Veljanovski et al. 2010) would be located within this branch (data not shown), showing the highest similarity to PvNTD9 and PvNTD11. Microarrays analysis in poplar demonstrates the important role of the acid phosphatase in the poplar defence mechanism against herbivores since this gene is one of the most strongly upregulated in response to wounding (Major and Constabel 2006).

The possible extracellular localization of the proteins encoded by the three genes induced by MeJA (Table 1) suggests that in response to methyl jasmonate, radicles would increase their ability to degrade nucleotides in the extracellular space. These nucleotides could come from the degradation of nucleic acids that are released into the environment when cells are damaged, or from the decomposition of organic matter in the soil. These enzymes could also participate in the maintenance of extracellular ATP homeostasis. This compound acts as a signalling molecule in plants, participating in functions as diverse as growth, development, and the response to abiotic and biotic stresses (Möhlmann et al. 2014). An eATP is released into the apoplastic space by exocytosis, damaged membrane or by specific transporter. In Arabidopsis, the eATP receptor has been identified (Choi et al. 2014) that gives rise to a signalling cascade in which eATP acts crosstalk with jasmonate, salicylic acid, and ethylene signalling pathways (Tripathi et al. 2018, Jewell et al. 2019). This cascade leads to downstream changes aimed to protect the plant against stresses and guaranteeing plant growth 
and development (Jewell et al. 2019, Pietrowska-Borek et al. 2020). The eATP content must be finely regulated to maintain cell physiology. The eATP is removed from the apoplast by the action of a set of enzymes, among which are e-nucleotidases, which are responsible for hydrolysing nucleotides, tri- and diphosphates, the 5'-nucleotidases, which degrade AMP giving rise to phosphate adenosine, and apoplastic nucleosidases that hydrolyse adenosine to adenine. Both molecules, adenosine and adenine, can be re-incorporated into cells via nucleoside transporters or purine permeases and thus recycled (Möhlmann et al. 2014). A model for the plant eATP signal transduction pathway with the involvement of $\mathrm{Ca}^{2+}$, reactive oxygen species, and MAP kinases among others has been recently proposed (Pietrowska-Borek et al. 2020). Interestingly, the promoter of the MeJA inducible genes is enriched in motif related to response to pathogenesis and several hormones including jasmonates, ethylene, and salicylic acid.

The nucleotidase activity in radicles from seedlings elicited with MeJA cannot rule out any of the hypotheses indicated for the induction in nucleotidase gene expression. The sequences of the members of the HAD superfamily are highly divergent, and the catalysed reactions and substrate specificities of each enzyme can only be determined empirically (Kuznetsova et al. 2006). The nucleotidase activity determined in the radicles increased with the treatment with methyl jasmonate regardless of the nucleotide tested, purine or pyrimidine, and of the $\mathrm{pH}$, acidic or neutral. The increase was not specific for nucleotides monophosphate, but it was also obtained for ADP and ATP. Therefore, the enzyme or enzymes induced by MeJA do not show great substrate specificity.

We describe here that MeJA induces nucleotide metabolism in common bean radicles. Among the identified gene candidates to code for nucleotidase, three of them induce its expression as far as after $24 \mathrm{~h}$ of MeJA elicitation in a dose-dependent manner. The induction of gene expression coincides with an increase in nucleotidase activity both at acidic and neutral $\mathrm{pH}$, and with all the nucleotides assayed, either purine or pyrimidine. It will be interesting to demonstrate if these genes are specifically induced in other adverse situations and to clearly identify the subcellular space where they show catalytic activity.

\section{References}

Armenteros, J.J.A., Sønderby, C.K., Sønderby, S.K., Nielsen, H., Winther, O.: DeepLoc: prediction of protein subcellular localization using deep learning. - Bioinformatics 33: 33873395, 2017.

Bogan, K.L., Brenner, C.: 5'-nucleotidases and their new roles in $\mathrm{NAD}^{(+)}$and phosphate metabolism. - New J. Chem. 34: 845853, 2010.

Bradford, M.M.: Rapid and sensitive method for quantitation of microgram quantities of protein utilizing principle of proteindye binding. - Anal. Biochem. 72: 248-254, 1976.

Burroughs, A.M., Allen, K.N., Dunaway-Mariano, D., Aravind, L.: Evolutionary genomics of the HAD superfamily: Understanding the structural adaptations and catalytic diversity in a superfamily of phosphoesterases and allied enzymes. - J. mol. Biol. 361: 1003-1034, 2006.

Cabello-Diaz, J.M., Quiles, F.A., Lambert, R., Pineda, M., Piedras, P.: Identification of a novel phosphatase with high affinity for nucleotides monophosphate from common bean (Phaseolus vulgaris). - Plant Physiol. Biochem. 53: 54-60, 2012.

Cabello-Díaz, J.M., Galvez-Valdivieso, G., Caballo, C., Lambert, R., Quiles, F.A., Pineda, M., Piedras, P.: Identification and characterization of a gene encoding for a nucleotidase from Phaseolus vulgaris. - J. Plant Physiol. 185: 44-51, 2015.

Camici, M., Garcia-Gil, M., Pesi, R., Allegrini, S., Tozzi, M.G.: Purine-metabolising enzymes and apoptosis in cancer. Cancers 11: 1354, 2019.

Choi, J., Tanaka, K., Cao, Y., Qi, Y., Qiu, J., Liang, Y., Lee, S.Y., Stacey, G.: Identification of a plant receptor for extracellular ATP. - Science 343: 290-294, 2014.

Clark, G., Roux, S.J.: Apyrases, extracellular ATP and the regulation of growth. - Curr. Opin. Plant Biol. 14: 700-706, 2011.

Dar, T.A., Uddin, M., Khan, M.M.A., Hakeem, K.R., Jaleel, H.: Jasmonates counter plant stress: a review. - Environ. exp. Bot. 115: 49-57, 2015.

Diaz-Baena, M., Galvez-Valdivieso, G., Delgado-Garcia, E., Pineda, M., Piedras, P.: Nuclease and ribonuclease activities in response to salt stress: identification of PvRNS3, a T2/Slike ribonuclease induced in common bean radicles by salt stress. - Plant Physiol. Biochem. 147: 235-241, 2020.

Duff, S.M.G., Sarath, G., Plaxton, W.C.: The role of acid phosphatases in plant phosphorus metabolism. - Physiol. Plant. 90: 791-800, 1994.

Edgar, R.C.: MUSCLE: multiple sequence alignment with high accuracy and high throughput. - Nucl. Acids Res. 32: 1792$1797,2004$.

Galvez-Valdivieso, G., Delgado-Garcia, E., Diaz-Baena, M., Montano, O., Quiles, F.A., Pineda, M., Piedras, P.: Biochemical and molecular characterization of PvNTD2, a nucleotidase highly expressed in nodules from Phaseolus vulgaris. - Plants 9: 171, 2020.

Gruber, B.D., Giehl, R.F.H., Friedel, S., Von Wiren, N.: Plasticity of the Arabidopsis root system under nutrient deficiencies. Plant Physiol. 163:161-179, 2013.

Haferkamp, I., Fernie, A.R., Neuhaus, H.E.: Adenine nucleotide transport in plants: much more than a mitochondrial issue. Trends Plant Sci. 16: 507-515, 2011.

Higo, K., Ugawa, Y., Iwamoto, M., Higo, H.: PLACE: a database of plant cis-acting regulatory DNA elements. - Nucl. Acids Res. 26: 358-359, 1998.

Huang, H., Liu, B., Liu, L.Y., Song, S.S.: Jasmonate action in plant growth and development. - J. exp. Bot. 68: 1349-1359, 2017.

Jewell, J.B., Sowders, J.M., He, R., Willis, M.A., Gang, D.R., Tanaka, K.: Extracellular ATP shapes a defense-related transcriptome both independently and along with other defense signaling pathways. - Plant Physiol. 179: 1144-1158, 2019.

Kumar, S., Stecher, G., Li, M., Knyaz, C., Tamura, K.: MEGA $\mathrm{X}$ : molecular evolutionary genetics analysis across computing platforms. - Mol. Biol. Evol. 35: 1547-1549, 2018.

Kuznetsova, E., Proudfoot, M., Gonzales, C.F., Brown, G., Omelchenko, M.V., Borozan, I., Carmel, L., Wolf, Y.I., Mori, H., Savchenko, A.V., Arrowsmith, C.H., Koonin, E.V., Edwards, A.M., Yakunin, A.F.: Genome-wide analysis of substrate specificities of the Escherichia coli haloacid dehalogenase-like phosphatase family. - J. biol. Chem. 281: 36149-36161, 2006.

Lambert, R., Cabello-Diaz, J.M., Quiles, F.A., Piedras, P.: 
Identification of nucleases related to nutrient mobilization in senescing cotyledons from French bean. - Acta Physiol. Plant. 38: 11, 2016.

Lambert, R., Quiles, F.A., Cabello-Diaz, J.M., Piedras, P.: Purification and identification of a nuclease activity in embryo axes from French bean. - Plant Sci. 224: 137-143, 2014.

Lambert, R., Quiles, F.A., Galvez-Valdivieso, G., Piedras, P.: Nucleases activities during French bean leaf ageing and darkinduced senescence. - J. Plant Physiol. 218: 235-242, 2017.

Livak, K.J., Schmittgen, T.D.: Analysis of relative gene expression data using real-time quantitative PCR and the 2(-Delta Delta C(T)) method. - Methods 25: 402-408, 2001.

Major, I.T., Constabel, C.P.: Molecular analysis of poplar defense against herbivory: comparison of wound- and insect elicitorinduced gene expression. - New Phytol. 172: 617-635, 2006.

Melino, V.J., Casartelli, A., George, J., Rupasinghe, T., Roessner, U., Okamoto, M., Heuer, S.: RNA catabolites contribute to the nitrogen pool and support growth recovery of wheat. - Front. Plant Sci. 9: 1539, 2018.

Möhlmann, T., Steinebrunner, I., Neuhaus, E.: Nucleotides and nucleosides: Transport, metabolism, and signaling function of extracellular ATP. - In: Lüttge, U., Beyschlag, W., Cushman, J. (ed.): Progress in Botany. Vol. 75, Pp. 119-144. Springer, Berlin - Heidelberg 2014.

Pietrowska-Borek, M., Dobrogojski, J., Sobieszczuk-Nowicka, E., Borek, S.: New insight into plant signaling: extracellular ATP and uncommon nucleotides. - Cells 9: 345, 2020.

Quiles, F.A., Galvez-Valdivieso, G., Guerrero-Casado, J., Pineda, M., Piedras, P.: Relationship between ureidic/amidic metabolism and antioxidant enzymatic activities in legume seedlings. - Plant Physiol. Biochem. 138: 1-8, 2019.

Quiles, F.A., Raso, M.J., Pineda, M., Piedras, P.: Ureide metabolism during seedling development in French bean (Phaseolus vulgaris). - Physiol. Plant. 135: 19-28, 2009.

Raza, A., Charagh, S., Zahid, Z., Mubarik, M.S., Javed, R., Siddiqui, M.H., Hasanuzzaman, M.: Jasmonic acid: a key frontier in conferring abiotic stress tolerance in plants. - Plant Cell Rep. early Access, 2020.
Saitou, N., Nei, M.: The neighbor-joining method: a new method for reconstructing phylogenetic trees. - Mol. Biol. Evol. 4: 406-425, 1987.

Seifried, A., Schultz, J., Gohla, A.: Human HAD phosphatases: structure, mechanism, and roles in health and disease. - FEBS J. 280: 549-571, 2013.

Soltabayeva, A., Srivastava, S., Kurmanbayeva, A., Bekturova, A., Fluhr, R., Sagi, M.: Early senescence in older leaves of low nitrate-grown Atxdh1 uncovers a role for purine catabolism in N supply. - Plant Physiol. 178: 1027-1044, 2018.

Todd, C.D., Tipton, P.A., Blevins, D.G., Piedras, P., Pineda, M., Polacco, J.C.: Update on ureide degradation in legumes. - J. exp. Bot. 57: 5-12, 2006.

Tripathi, D., Zhang, T., Koo, A.J., Stacey, G., Tanaka, K.: Extracellular ATP acts on jasmonate signaling to reinforce plant defense. - Plant Physiol. 176: 511-523, 2018.

Untergasser, A., Nijveen, H., Rao, X., Bisseling, T., Geurts, R., Leunissen, J.A.: Primer3Plus, an enhanced web interface to Primer3. - Nucl. Acids Res. 35: W71-W74, 2007.

Veljanovski, V., Major, I.T., Patton, J.J., Bol, E., Louvet, S., Hawkins, B.J., Constabel, C.P.: Induction of acid phosphatase transcripts, protein and enzymatic activity by simulated herbivory of hybrid poplar. - Phytochemistry 71: 619-626, 2010.

Wang, J., Song, L., Gong, X., Xu, J.F., Li, M.H.: Functions of jasmonic acid in plant regulation and response to abiotic stress. - Int. J. mol. Sci. 21: 1446, 2020.

Wasternack, C., Strnad, M.: Jasmonates: news on occurrence, biosynthesis, metabolism and action of an ancient group of signaling compounds. - Int. J. mol. Sci. 19: 2539, 2018.

Watanabe, S., Matsumoto, M., Hakomori, Y., Takagi, H., Shimada, H., Sakamoto, A.: The purine metabolite allantoin enhances abiotic stress tolerance through synergistic activation of abscisic acid metabolism. - Plant Cell Environ. 37: 1022-1036, 2014.

Zrenner, R., Stitt, M., Sonnewald, U., Boldt, R.: Pyrimidine and purine biosynthesis and degradation in plants. - Annu. Rev. Plant Biol. 57: 805-836, 2006. 